\title{
Systemic drug related intertriginous and flexural exanthema (SDRIFE) or intertriginous drug eruption: A matter of semantics
}

\section{Dhaarna Wadhwa, Vikram K Mahajan}

\author{
Department of Dermatology, Venereology \& Leprosy, Dr. R. P. Govt. Medical College, Kangra (Tanda)-176001 \\ (Himachal Pradesh), India
}

Corresponding author: Dr. Vikram K Mahajan, E-mail: vkm1@rediffmail.com

\begin{abstract}
Systemic drug-related intertriginous and flexural exanthema (SDRIFE) is a recently coined term to describe an uncommon adverse cutaneous drug reaction described previously as intertriginous drug eruption or flexural drug exanthema. Characteristic flexural erythema notably of the axillae and groins following systemic administration of offending drug may eventuate to TEN-like eruption with relative absence of systemic involvement. Beta-lactam antibiotics like amoxicillin remain the most common offending drug. Al-year-old boy developed SDRIEF overnight after oral amoxicillin $(125 \mathrm{mg})$ given for upper respiratory infection. The eruptions deteriorated evolving to TEN-like skin tenderness and exfoliation despite withdrawal of offending drug and treatment with oral cetirizine and prednisolone. It subsided within 1-2days after intravenous immunoglobulin treatment. The putative drugs and various pathogenetic mechanisms proposed for this very unusual adverse cutaneous drug reaction reflects that the nomenclature SDRIFE, intertriginous drug eruption or flexural drug exanthema is just semantics.
\end{abstract}

Key words: Amoxicillin; Baboon syndrome; Flexural drug eruption; Intertriginous drug eruption; SDRIFE;

Systemic contact dermatitis

\section{INTRODUCTION}

The term 'baboon syndrome' was first described in 1984 by Andersen to describe a mild systemic cutaneous reaction characterized by diffuse bright red erythema resembling red bottoms of baboons after oral exposure to allergens such as nickel, mercury or drugs [1]. The "symmetrical drug related intertriginous and flexural exanthema" (acronym; SDRIFE) is a recent term coined by Häusermann et al [2] in 2004 for a similar entity induced by systemic drugs that was invariably described as 'systemically induced allergic contact dermatitis', 'systemic contact dermatitis', 'drug-induced intertrigo', 'eczema rubrum', 'flexural drug eruption', 'intertriginous drug eruption' or 'paraptic eczema'. It is an unusual yet characteristic drug-related eruption that typically involves the flexural folds and gluteal areas without any predilection for age or gender. Clinically, well delineated erythema of the perigenital and perianal area and maculopapular eruptions involving the large folds such as the neck-fold, axillae, cubital fossae and groins appears within hours to 2 days of drug exposure. Palms, soles, face and mucosae are usually spared. Rarely the lesions may consist of pustules, vesicles or bullae. Systemic features like fever, lymphadenopathy and internal organ involvement usually do not occur [2-4]. The most common offending drugs are antibiotics, especially beta-lactams (benzylpenicillin, phenoxymethylpenicillin, ceftriaxone, cefuroxime, and ceftazidine) with amoxicillin topping the list. The other implicated drugs include pseudoephedrine, roxithromycin, allopurinol, barium sulfate-containing contrast media, omeprazole, hydroxyzine, cimetidine, deflazacort, hydroxyurea, heparin, immunoglobulin, mitomycin $\mathrm{C}$, naproxen, oxycodone, terbinafine, cetuximab, valacyclovir, risperidone, and very rarely clindamycin [2,5-8].

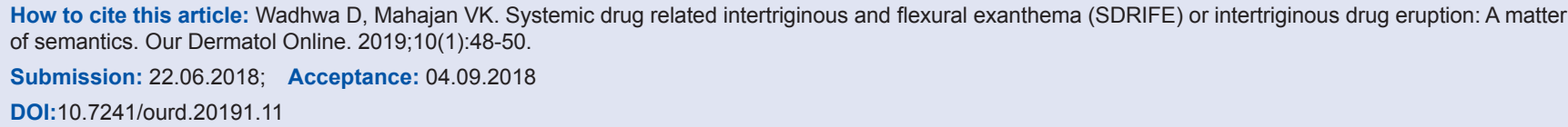




\section{CASE REPORT}

Al-year-old boy developed a symmetric, confluent, erythematous, mildly pruritic, and macular eruptions affecting both axillae and inguinal folds overnight after oral amoxicillin (125mg) given for upper respiratory infection. Similar lesions over the cubital fossa, buttocks and the lower abdomen appeared during next 1-2 days. Parents denied using any topical treatment earlier. His family and past medical history was unremarkable, he was immunized for his age and weighed $10 \mathrm{~kg}$. He was uncomfortable, irritable, and has no fever, pallor, lymphadenopathy or other systemic disease. A review of his laboratory parameters for complete blood counts, hepato-renal function tests, urinalysis, chest $\mathrm{x}$-ray and electrocardiogram revealed no abnormality. No fungal hyphae were visualized in $\mathrm{KOH}$ mounts from skin lesions. Parents did not consent for skin biopsy. A diagnosis of SDRIFE was made based on clinical criteria (Tablel) proposed by Hausermann et al. [2] . Amoxicillin was stopped and he was prescribed oral cetirizine $5 \mathrm{mg} / \mathrm{d}$. Oral prednisolone $10 \mathrm{mg} / \mathrm{d}$ was added when his irritability, skin tenderness and eruptions deteriorated to TEN-like exfoliation particularly over flexurals but without benefit (Fig. 1). At this point

Table 1: Clinical diagnostic criteria for systemic drug-related intertriginous and flexural exanthema (SDRIFE)

\begin{tabular}{|c|c|}
\hline S. No & Diagnostic criteria \\
\hline 1. & $\begin{array}{l}\text { Exposure to a systemically administered drug either following the } \\
\text { first or subsequent dose (excluding contact allergens) }\end{array}$ \\
\hline 2. & $\begin{array}{l}\text { Sharply demarcated erythema of the gluteal/perianal area and/or } \\
\text { V-shaped erythema of the inguinal/perigenital area }\end{array}$ \\
\hline 3. & Involvement of at least one other intertriginous/flexural localization \\
\hline 4. & Symmetry of affected areas \\
\hline 5. & Absence of systemic symptoms and signs \\
\hline
\end{tabular}

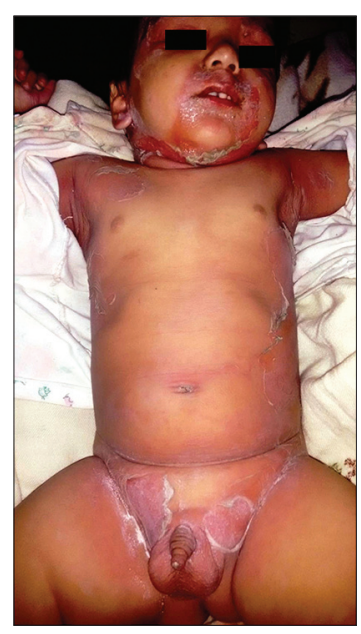

Figure 1: Erythematous and TEN-like exfoliating skin rash predominantly involving periocular and perioral skin, and neck, axillary and inguinal folds. treatment with intravenous immunoglobulin (IVIG, $2.5 \mathrm{gm} /$ day for 4 days) initiated. His eruptions resolved over next 1- 2 days without any residual pigmentation or scarring. Parents did not consent for oral re-challenge and were advised to avoid amoxicillin in future.

\section{DISCUSSION}

As in our patient the diagnosis of SDRIFE remains clinical in most cases not requiring extensive investigative work up. On the other hand, histologically it is characterized by superficial perivascular infiltrate primarily of mononuclear cells and in some cases neutrophils and eosinophils. Rarely, there may be EM- or TEN-like histologic features of necrotic keratinocytes with vacuolar and hydropic degeneration of the basal cell layer [2]. Drug re-challenge is diagnostic but has potential to trigger severe reactions. Patch and prick tests have low sensitivity and diagnostic value. The utility of in-vitro lymphocyte transformation test that may detect the T-cell mediated delayed hypersensitivity response, macrophage migration or indirect rat mast cell degranulation tests remains limited as a research tool [6]. SDRIFE carries a good prognosis with complete resolution following withdrawal of offending drug. Topical corticosteroids and systemic antihistamines may provide symptomatic relief. Although severe form has been treated with systemic corticosteroids, our patients recovered only after IVIG administration that apparently is more effective than corticosteroids [9].

Several pathomechanisms for this baffling entity have been proposed in last few years. It was initially speculated to be a type IV delayed hypersensitivity to the offending drug involving macrophages and Thl response (type IVa reaction) and cytotoxic CD8 T cells (type IVc reaction) [2,4]. Daito et al [7] and Wolf et al [10] considered it a type of recall phenomenon means a recall of previous dermatitis such as diaper dermatitis in childhood. However, the concept as proposed by Mahajan et al [9] appears more plausible. It suggests that the offending drug or its metabolites first get concentrated in the apo-eccrine glands preferentially localized to flexural skin and produce a direct toxicity to them. The concentrated drug/ metabolites then gets excreted on the skin surface in a gradient fashion, highest concentration in the upper epidermal layers than in the deeper layers, and inflicts a non-immunologic direct toxicity and necrosis of keratinocytes in outer layers while occlusion, sweating 
and friction aggravate it further. Their hypothesis has also been ratified in a recently proposed $\mathrm{p}-\mathrm{i}$ (pharmacologic interaction with immunoreceptors) concept by Miyahara et al. [5] According to them, certain drugs are able to bind directly and noncovalently to T-cell receptors without first being presented by major histocompatibility complex (MHC) molecules and without prior metabolism (direct recognition).However, the putative drugs and various pathogenetic mechanisms proposed for this very unusual adverse cutaneous drug reaction reflects that the nomenclature SDRIFE, intertriginous drug eruption or flexural drug exanthema is just semantics.

\section{CONSENT}

The examination of the patient was conducted according to the Declaration of Helsinki principles.

\section{REFERENCES}

1. Andersen KE, Hjorth N, Menne T. The baboon syndrome: systemically-induced allergic contact dermatitis. Contact Dermatitis 1984;10:97-100.

2. Häusermann P, Harr T, Bircher AJ. Baboon syndrome resulting from systemic drugs: is there strife between SDRIFE and allergic contact dermatitis syndrome? Contact Dermatitis. 2004;51:297-310.

3. Häusermann P, Bircher AJ. SDRIFE - another acronym for a distinct cutaneous drug exanthema: do we really need it? Dermatology. 2007;214:1-2.

4. ÖzkayaE, Babuna G. A challenging case: symmetrical drug related intertriginous and flexural exanthem, fixed drug eruption, or Both? Pediatr Dermatol. 2011;28:711-4.

5. Miyahara A, Kawashima H, Okubo Y, Hoshika A. A new proposal for a clinical-oriented sub classification of baboon syndrome and a review of baboon syndrome. Asian Pac J Allergy Immunol. 2011;29:150-60.

6. Kardaun SH, Tupker RA. Symmetric drug-related intertriginous and flexural exanthema (Baboon syndrome) induced by omeprazole. Int J Dermatol. 2012;51:1134-7.

7. Daito J, Hanada K, Katoh N, Katoh S, Sakamoto K, Asai J, et al. Symmetrical drug-related intertriginous and flexural exanthema caused by valacyclovir. Dermatology. 2009;218:60-2.

8. Mahajan VK, Sharma RC. Intertriginous drug eruptions. Indian J Dermatol. 2005;50:146-9.

9. Mahajan VK, Sharma NL, Jindal R. Intertriginous drug eruption: report of a case and proposed pathogenetic mechanism. Int J Dermatol. 2008;47:1310-1.

10. Wolf R, Orion E, Matz H. The baboon syndrome or intertriginous drug eruption: a report of eleven cases and a second look at its pathomechanism. Dermatol Online J. 2003;9:2.

Copyright by Dhaarna Wadhwa and Vikram $\mathrm{K}$ Mahajan. This is an open-access article distributed under the terms of the Creative Commons Attribution License, which permits unrestricted use, distribution, and reproduction in any medium, provided the original author and source are credited.

Source of Support: Nil, Conflict of Interest: None declared. 